Cahiers $d u$ MONDE RUSSE

\section{Cahiers du monde russe}

Russie - Empire russe - Union soviétique et États indépendants

50/2-3 | 2009

L'Europe orientale, 1650-1730. Crises, conflits et renouveau

\title{
Modernism and Public Reform in Late Imperial RussiaIlya GUERASIMOV
}

, New York : Palgrave Macmillan, 2009, 325 p.

\section{Alessandro Stanziani}

\section{OpenEdition}

\section{Journals}

Édition électronique

URL : https://journals.openedition.org/monderusse/9751

DOI : 10.4000/monderusse. 9751

ISSN : $1777-5388$

\section{Éditeur}

Éditions de l'EHESS

\section{Édition imprimée}

Date de publication : 15 septembre 2009

ISBN : 978-2-7132-2260-3

ISSN : $1252-6576$

\section{Référence électronique}

Alessandro Stanziani, " Modernism and Public Reform in Late Imperial Russiallya GUERASIMOV » Cahiers du monde russe [En ligne], 50/2-3 | 2009, mis en ligne le 14 janvier 2013, consulté le 03 septembre 2022. URL : http://journals.openedition.org/monderusse/9751 ; DOI : https://doi.org/ 10.4000/monderusse. 9751

Ce document a été généré automatiquement le 3 septembre 2022.

Tous droits réservés 


\title{
Modernism and Public Reform in Late Imperial RussiaIlya GUERASIMOV
}

, New York : Palgrave Macmillan, 2009, 325 p.

\author{
Alessandro Stanziani
}

\section{Ilya GUERASIMOV, Modernism and Public Reform in Late Imperial Russia, Rural Professionals and Self-Organization, 1905-1930, New York : Palgrave Macmillan, 2009, 325 p.}

1 Ilya Guerasimov est l'un des rédacteurs de $A b$ Imperio qu'un groupe de jeunes chercheurs établis à Kazan' a rapidement réussi à imposer à l'attention internationale, au cours de la dernière décennie, comme l'une des revues les plus novatrices sur la Russie et l'exEmpire soviétique. D'origine bulgare, il a commencé à travailler voici une bonne dizaine d'années sur Aleksandr V. Čajanov, ce spécialiste de l'agronomie russe, actif au début du $\mathrm{xx}^{\mathrm{e}}$ siècle et qui a si fortement influencé les études rurales et le mouvement coopératif dans le monde entier, surtout à partir des années 1960. Guerasimov lui a consacré sa thèse de doctorat et un ouvrage en russe (Duša človeka perehodnogo vremeni, 1997). Il a décidé ensuite d'étudier l'ensemble d'une génération de spécialistes agraires en Russie entre 1905 et 1930, date à laquelle ceux-ci furent massivement emprisonnés et écartés de tout poste de responsabilité. Ce livre est le résultat de ces recherches. L'auteur vise à rendre compte de la formation, de l'insertion professionnelle, sociale et politique de ces spécialistes ainsi que de leur impact sur la paysannerie. L'argument principal est qu'en dépit d'une tendance de l'intelligentsia russe à toujours évoquer le « peuple » sans avoir réellement de liens avec lui, cette génération de spécialistes a réellement su établir un "pont" avec les paysans. Une véritable croissance économique, accompagnée d'un développement social considérable, vit dès lors le jour en Russie, évolution que la Première Guerre mondiale, puis la révolution bolchevique allaient entraver puis stopper définitivement. 
2 L'ouvrage se compose de onze chapitres répartis en trois parties. La première, intitulée "Structures de la mobilisation", étudie les fondements sociaux et structurels qui ont permis l'émergence du mouvement social généré par la "génération Čajanov ». Le premier chapitre tente d'identifier les paramètres et les catégories interprétatives des réformateurs russes; il examine en particulier la diffusion des journaux et périodiques agricoles dans les campagnes, l'intérêt de l'intelligentsia pour l'agriculture, les influences américaines et allemandes sur ce milieu. Le deuxième est consacré à la formation des spécialistes agraires et montre comment le développement des instituts, écoles et universités offrant des formations dans ce domaine entraîna la constitution d'une génération authentiquement nouvelle - tant sur le plan démographique, politique, qu'intellectuel. Le troisième chapitre détaille la manière dont ces spécialistes voulurent contribuer à l'éducation et à la formation professionnelle des paysans en s'inspirant notamment de la méthode des « chaires itinérantes » d'agronomie italiennes, sortes d'universités populaires " mobiles » se déplaçant dans les campagnes.

3 La deuxième partie - « Dynamiques de la modernisation " - analyse l'interaction entre ces spécialistes et les autres groupes sociaux. Le chapitre IV étudie l'évolution de l'État tsariste, les réformes agraires (zemstva et coopératives) ainsi que l'attitude de ces différents milieux face à ces réformes. La section suivante propose une analyse fine du marché du travail des spécialistes, c'est-à-dire la façon dont, sur le terrain, ambitions et projets personnels ont pu se concrétiser. Puis l'auteur détaille les réactions des paysans en soulignant la forte convergence entre ces derniers et les spécialistes. Le chapitre VII fait état de la crise de l'agronomie sociale à la veille de la Première Guerre mondiale, victime, selon l'auteur, de son succès, et décrit les tensions irrésolues entre radicalisme politique et professionnalisme qui en ont résulté.

4 Les « sentiers de la nationalisation " (troisième partie) ajoutent une dimension nouvelle à une histoire déjà complexe, celle de la diversité des expériences locales dans le contexte impérial russe. La combinaison de ces différents niveaux se traduit finalement par des points de vue assez contrastés entre nationalisme, autonomie, revendications locales et ambitions supranationales des agronomes. Le chapitre VIII met ainsi en lumière comment ces derniers avaient pour la plupart développé un fort sentiment nationaliste panrusse, surtout au début de la Première Guerre mondiale. Guerasimov étudie dans ce contexte les attitudes hétérogènes et fluctuantes envers la pensée "étrangère ». Puis il poursuit cette investigation en montrant l'impact de la mobilisation sur les agronomes, mais aussi les réactions locales, voire nationalistes, surtout en Ukraine. Le chapitre X se penche sur l'impact des révolutions de 1917 et note la résurgence de tendances au durcissement chez certains agronomes qui semblent renier leurs doctrines antérieures opposant leur "professionnalisme" au radicalisme des générations précédentes. L'échec de la révolution de Février, puis celui de leurs ambitions à la suite de la révolution d'Octobre montre, selon l'auteur, l'erreur de cette génération qui avait renoncé à ses orientations politiques et professionnelles. Le dernier chapitre conclut sur sa disparition progressive dans l'URSS postrévolutionnaire.

5 Malgré sa complexité, l'ouvrage offre un panorama clair, raisonné et en partie nouveau d'une génération d'agronomes qui a constitué un élément important au sein de la société russe puis soviétique, mais a également influencé les programmes de développement agraire sur plusieurs continents tout au long $\mathrm{du} \mathrm{xx}^{\mathrm{e}}$ siècle. Fruit de longues années d'études, il constitue désormais une référence incontournable pour tous ceux qui s'intéressent à ces problématiques. En même temps, il suscite des interrogations tant sur 
le sujet lui-même que sur la manière dont il est traité. Passons sur le fait que l'auteur se comporte comme si personne avant lui n'avait étudié ce domaine - cela pourrait à la limite s'expliquer, et encore, pour les études en langue française sans doute moins présentes dans les circuits intellectuels reliant Kazan' aux États-Unis -, mais c'est plus difficile à justifier pour les travaux russes et anglo-saxons, très nombreux sur ce sujet et qui sont presque entièrement ignorés (notamment les ouvrages de Yanni Kotsonis et de E. Kingston-Mann). L'auteur se dispense par là de montrer en quoi son analyse se distingue de toutes celles qui l'ont précédée.

Concernant le contenu, quid du lien entre professionnalisme et orientation politique des spécialistes agraires? Guerasimov prend au pied de la lettre les affirmations de certains spécialistes de la "génération Čajanov » opposant leur professionnalisme à l'idéologie des révolutionnaires et populistes de la génération précédente et à celle des agronomes d'État. Sans doute, cette opposition avait-elle un sens; cependant, comment oublier que derrière l'argument du professionnalisme, donc de la priorité des compétences, ces auteurs visaient aussi à critiquer des orientations politiques qu'ils considéraient comme extrêmes, comme la redistribution révolutionnaire des terres et la commune, d'une part, et la privatisation des terres, de l'autre? En d'autres termes, nous estimons que, plutôt que de reproduire tels quels les arguments des agronomes, il aurait mieux valu, au lieu de les opposer, mettre en évidence l'imbrication étroite, dans la Russie de l'époque, entre orientations politiques et professionnelles. Ce que fait d'ailleurs l'auteur par moments en soulignant l'importance de la notion de obščestvennost' (engagement social) pour la plupart de ces acteurs.

7 À partir de là, le recours à la notion de génération, que Guerasimov emprunte à Karl Mannheim, devient problématique. Car il s'agit là moins de "génération " au sens démographique du terme que d'un ensemble d'éléments: orientations politiques, formation, insertion sociale, propres à un groupe donné et à une époque donnée. Cette suggestion est pertinente ; encore aurait-il fallu, afin de pouvoir la justifier, comparer en détail la génération Čajanov à une autre génération au minimum, sans quoi il est difficile de parler de "génération" au sens de Mannheim. Ce point est important car, en soulignant trop l'écart (non démontré) avec la génération précédente, Guerasimov reprend sans réserve l'argument du groupe qu'il étudie et que ces auteurs avancent à un moment particulier, avant la Première Guerre mondiale, lorsqu'ils veulent se démarquer des partis socialistes et révolutionnaires. Cependant, cet argument politique et rhétorique dissimulait les liens que plusieurs d'entre eux entretenaient avec ces mêmes partis et avec les acteurs de la génération précédente. Cet élément aurait peut-être évité à l'auteur d'être surpris par le regain de radicalisme des « Tchayanoviens » à l'époque de la révolution.

8 La troisième partie est sans doute la plus novatrice, du moins par rapport au sujet traité que les travaux existants ont souvent limité au cas spécifiquement russe. En mettant l'accent sur les tensions entre dimension locale, nationale, impériale et supranationale, Guerasimov touche à un aspect qui mériterait assurément d'être plus développé. Ce jeu d'échelles multiples sur le savoir au sens large du terme (éducation générale et professionnelle, sciences exactes et de la société, etc.) au $\mathrm{xx}^{\mathrm{e}}$ siècle se limite trop souvent à une opposition entre dimensions nationale (voire nationaliste) et internationale. C'est là une piste que nous espérons voir développée, et pourquoi pas, par Guerasimov luimême. 